THEORY OF DIFFUSIONLESS PHASE TRANSITIONS

\author{
By \\ Richard James \\ and \\ David Kinderlehrer
}

IMA Preprint Series \# 492

February 1989 


\title{
Theory of Diffusionless Phase Transitions
}

\author{
Richard James \\ Department of Aerospace Engineering and Mechanics \\ University of Minnesota \\ Minneapolis, Minnesota \\ David Kinderlehrer \\ School of Mathematics \\ University of Minnesota \\ Minneapolis, Minnesota
}

\section{Introduction}

The aim of this report is to explore a constitutive theory able to predict macroscopic state functions and the detailed microstructure of a crystal which suffers a diffusionless solid-solid transformation involving a change in symmetry. A principal feature of this theory is that we are led to consider energy functionals which fail to be lower semi-continuous, with respect to an appropriate notion of weak convergence. In these circumstances, the infimum of energy may be achieved only in some generalized sense while a minimizing sequence may develop successively finer oscillations reminiscent of a finely twinned microstructure. 1,2

To understand very briefly the consequences of material symmetry in this regard, let us consider an energy density $W(F)$ defined for an elastic body in three dimensional space at some fixed temperature. The condition of frame indifference is that

$$
\mathrm{W}(\mathrm{QF})=\mathrm{W}(\mathrm{F}) \quad \text { for } \quad \mathrm{Q}^{\mathrm{T}} \mathrm{Q}=1, \operatorname{det} \mathrm{Q}=1 \text {, and } \operatorname{det} \mathrm{F}>0 \text {. }
$$

The variant structure of certain crystals, for example, many ionic solids, shape memory alloys, and ferroelectrics, suggests that coexistent symmetry related phases may be present in an configuration. An example of this is twinning. A deformation $A$ is symmetry related to $F$ provided

1 This research was supported under NSF MSM 8612420 and by the NSF and ASOFR under DMS 871881

2 Proceedings of Equations a Derivées Partielles et Modeles Continus de Transitions de Phases, Nice 1988 


$$
\mathrm{A}=\mathrm{QFH}
$$

for some proper rotation $\mathrm{Q}$ and some $\mathrm{H}$ in a given "symmetry group" and

$$
\mathrm{W}(\mathrm{A})=\mathrm{W}(\mathrm{F}) \text {. }
$$

The two variants coexist in a configuration when there is a piecewise linear deformation $y(x)$ with, say,

$$
\nabla y(x)=\left\{\begin{array}{ll}
F & \text { if } \quad x \cdot n<0 \\
A & \text { if } x \cdot n>0
\end{array},\right.
$$

for some unit vector $n \in \mathbb{R}^{3}$. This is only possible if $\operatorname{rank}(A-F)=1$, provided $A \neq F$, as Hadamard pointed out, and in fact,

$$
A=F+\alpha \otimes n \quad \text { for some nonzero } \alpha \in \mathbb{R}^{3} \text {. }
$$

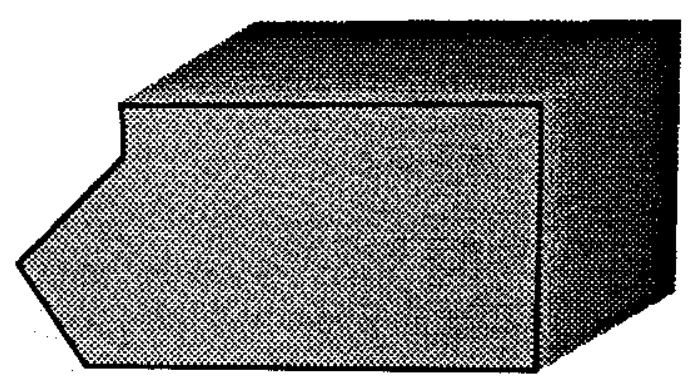

Fig. 1 Schematic drawing of a single crystal

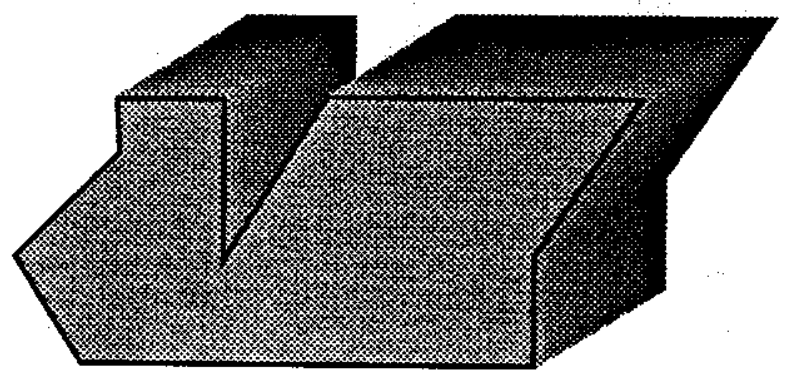

Fig. 2 Schematic drawing of a twinned crystal 


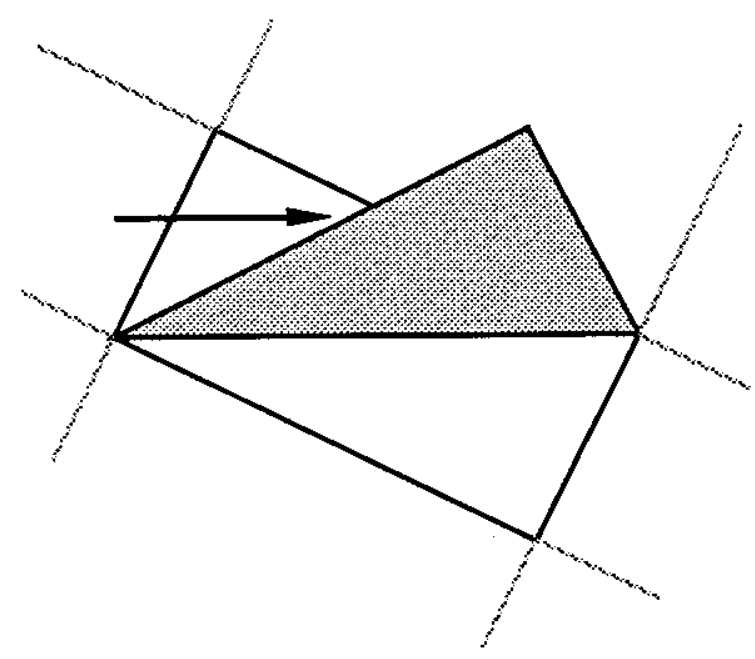

Fig 3. Illustration of the motion of a lattice element on the twin plane according to (1.4). The upper triangle undergoes simple shear which renders its deformed state congruent, in three dimensions, to its undeformed state.

Suppose, for clarity, that $W(F)=\min W=0$. Then the function $f(t)=$ $\mathrm{W}(\mathrm{F}+\mathrm{t} \alpha \otimes n)$ vanishes together with its derivative at $t=0$ and $t=1$ and thus at some $\lambda \in$ $(0,1)$,

$$
\mathrm{f}^{\prime \prime}(\lambda)=\frac{\partial^{2} W}{\partial \mathrm{A}_{\mathrm{ij}} \partial \mathrm{A}_{h \mathbf{k}}} \alpha^{\mathrm{i}} \alpha^{\mathrm{h}_{\mathrm{n}} \mathrm{j}_{\mathrm{n}} \mathrm{k}}<0
$$

unless $f$ is constant.

Thus the familiar Legendre-Hadamard condition is violated for $W$. Of course, we know that lower semi-continuity of the functional

$$
E(v)=\int_{\Omega} w(\nabla v) d x
$$

implies the Legendre-Hadamard condition, cf. Morrey [51]. So our ability to understand variant structures in crystalline solids depends on our willingness to study functionals which are not lower-semicontinuous.

On the other hand, let us write

$$
A=F(1+a \otimes n) .
$$


Given $\lambda \in(0,1)$, introduce the characteristic function $\chi(t)$ of the interval $(0, \lambda) \subset(0,1)$, extended periodically to $(-\infty, \infty)$, so $\chi(t)=\chi(t+k), k \in Z$. Let

$$
u^{k}(x)=F\left(x+\frac{1}{k} \int_{0}^{k x \cdot n} \chi(t) d t a\right)
$$

so that

$$
\begin{aligned}
\mathrm{F}^{\mathrm{k}}(\mathrm{x}) & =\nabla_{\mathrm{u}^{\mathrm{k}}}(\mathrm{x})=\mathrm{F}(1+\chi(\mathrm{kx} \cdot \mathrm{n}) \mathrm{a} \otimes \mathrm{n}) \\
& =\left\{\begin{array}{rl}
\mathrm{F} & \text { if } \chi=0 \\
\mathrm{~A} & \text { if } \chi=1
\end{array} .\right.
\end{aligned}
$$

Now the ( $\mathrm{F}^{\mathrm{k}}$ ) do not converge in a usual pointwise sense, but they do converge in the weak* topology. Indeed,

$$
\mathrm{F}^{\mathbf{k}} \rightarrow(1-\lambda) \mathrm{F}+\lambda \mathrm{A} \quad \text { in } \mathrm{L}^{\infty}(\Omega) \text { weak*, }
$$

when $\Omega \subset \mathbb{R}^{3}$ is bounded, since the sequence of periodic functions $\chi(k t), 0 \leq t \leq 1$, converges weak* to its mean value $\lambda$. Inspection of the ( $F^{k}$ ) shows that it represents a sequence of twinned states with successively finer structure. From the viewpoint of energy,

$$
\begin{aligned}
W(F k(x)) & =\left\{\begin{array}{lll}
W(F) & \text { if } \chi=0 \\
W(A) & \text { if } \chi=1
\end{array}\right. \\
& =0, \text { for all } k
\end{aligned}
$$

We see in this way that the macroscopic limit deformation, which itself is not a minimizer of energy, is obtained by successive shearing of minimum energy configurations. In the sequel we shall illustrate how the energy of the limit configuration may be interpreted as $\lim _{x \rightarrow \infty} W(F)=$ 0 by use of the parametrized measure or Young measure [74], cf. also [67]. This measure serves as an accounting device to record the oscillations of the process through which the limit configuration is achieved. 


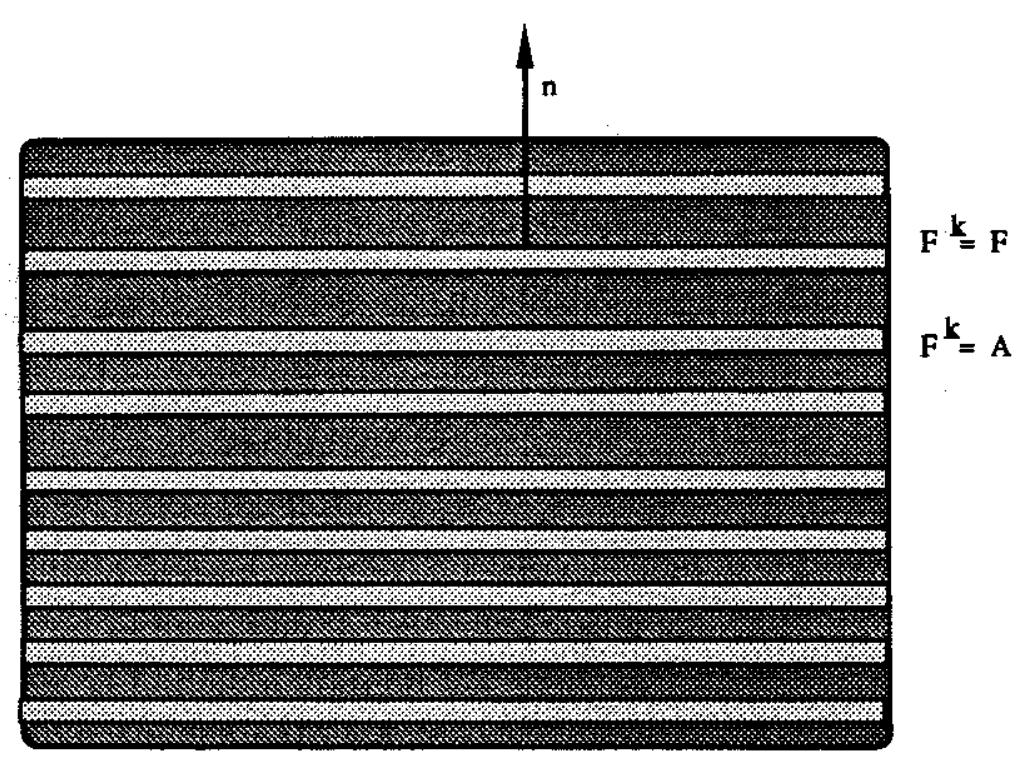

Fig. 4 Illustration of fine phase behavior, as given by $F^{*}$ in (1.9).

The phenomenological theory we are about to describe has its origins in the work of Ericksen [21-29]. Our idea of equilibrium follows from Gibbs [34]. Important contributions are due to Gurtin [35], Müller [52], Müller and Wilmanski [53], Parry [54], and Pitteri [61-63]. Some of our thoughts about phase transitions have been motivated by studying $[10,12,16,20,71,72,73]$. This report is based primarily on [6],[14]. The paper by Ball [5] has some connections with this one. Computational work related to the theory and analysis presented here has been undertaken by Collins and Luskin [17]. Other work of possible interest to the reader includes $[38-41,42,43]$.

\section{Constitutive equations}

We now describe how particular energy density functions $W(F, \theta)$ are obtained. Sometimes we shall depress the dependence of the energy on temperature $\theta$. We begin with lattice considerations. Our idea of a crystal is that it consists of a regular lattice consisting of identical atoms, molecules, or more general symmetry elements $L\left(e_{1}, e_{2}, e_{3}\right)$ determined by linearly independent lattice vectors $\left[\mathrm{C}_{i}\right)$ according to the rule

$$
L\left(\mathrm{e}_{1}, \mathrm{e}_{2}, \mathrm{e}_{3}\right)=\left\{\mathrm{x} \in \mathbf{R}^{3}: \mathrm{x}=\mu^{\mathrm{i}} \mathrm{e}_{\mathrm{i}}, \mu^{\mathrm{i}} \in \mathbf{Z}\right\} .
$$


The summation convention is understood to be in force. A common procedure involves assigning a central force potential and then summing the contributions of all the energies due to pairs of atoms in a large volume. The temperature dependence of the energy density is then calculated by recognizing that the atoms vibrate about the definite positions in $L\left(e_{1}, e_{2}, e_{3}\right)$. Standard statistical arguments then provide a temperature correction. It is well known that this procedure fails in that it predicts the Cauchy relations which are not satisfied by many materials of interest in studies of phase transformations. As discussed by Christian [16, Chapter 5] modem attempts to improve the calculation have been largely unsuccessful, and, in fact, many of these calculations once again give back the Cauchy relations!

Recognizing that for numerous calculations of the type illustrated in the introduction we will not need the detailed form of $\mathrm{W}$, we adopt a more modest but general point of view. A derivation of $\mathrm{W}$ from first principles will have the property that once the positions of the lattice elements are known, the energy density is determined. Following this line of thought, we postulate the existence of $\Phi\left(\mathrm{e}_{1}, \mathrm{e}_{2}, \mathrm{e}_{3}, \theta\right)$ which represents the energy per unit volume of a lattice $L\left(\mathrm{e}_{1}, \mathrm{e}_{2}, \mathrm{e}_{3}\right)$ at temperature $\theta$. The danger in this procedure is that two different sets of lattice vectors may generate the same lattice, i.e., the same atomic positions. This possibility is addressed by a classical theorem in crystallography, cf. [24 ] or [29], stating that the energy does not depend on the choice of lattice basis, leading to the invariance statement

$$
\Phi\left(\mu_{1}^{j} \mathrm{e}_{j}, \mu_{2}^{j} \mathrm{e}_{j}, \mu_{3}^{\mathrm{j}} \mathrm{e}_{j}, \theta\right)=\Phi\left(\mathrm{e}_{1}, \mathrm{e}_{2}, \mathrm{e}_{3}, \theta\right) \quad \text { for all } \mathbf{M}=\left(\mu_{i}^{\mathrm{j}}\right) \in \mathbf{Z}^{9}, \operatorname{det} \mathbf{M}= \pm 1 .(2.2)
$$

We may pass to a continuum theory by adopting the Cauchy - Born Rule $[24,27]$. For this, we fix a basis of reference lattice vectors $\left\{e_{i}^{0}\right\}$ and define

$$
\mathrm{W}(\mathrm{F}, \theta)=\frac{\left(\mathrm{Fe}_{1}^{0} \wedge \mathrm{Fe}_{2}^{\mathrm{o}}\right) \cdot \mathrm{Fe}_{3}^{\mathrm{o}}}{\left(\mathrm{e}_{1}^{0} \wedge \mathrm{e}_{2}^{\mathrm{o}}\right) \cdot \mathrm{e}_{3}^{\mathrm{o}}} \Phi\left(\mathrm{Fe}_{1}, \mathrm{Fe}_{2}, \mathrm{Fe}_{3}, \theta\right), \quad \operatorname{det} \mathrm{F}>0
$$

The factor in front of $\Phi$ merely converts the energy per unit volume to an energy per unit reference volume to be more consistent with standard continuum mechanics. This factor is really just det $F$. With this rule, the $\Omega$ introduced in $\S 1$ becomes identified with the lattice $L\left(\mathrm{e}_{1}^{\mathrm{o}}, \mathrm{e}_{2}^{\mathrm{o}}, \mathrm{e}_{3}^{\mathrm{o}}\right)$.

Clearly $W$ should be frame indifferent at each fixed $\theta$ and so satisfy (1.1). As well, it inherits the invariance from (2.2) 


$$
\begin{aligned}
& W(F H, \theta)=W(F, \theta), \quad \text { for all } H \in G\left(\left\{e_{i}^{o}\right\}\right) \text {, where } \\
& G\left(\left\{e_{i}^{o}\right\}\right)=E^{o} G L\left(Z^{3}\right)\left(E^{o}\right)^{-1},
\end{aligned}
$$

where $E^{\circ}$ is the matrix whose columns are $\left\{e_{i}^{0}\right\}$. Note that if $F_{o}$ minimizes $W$ at some fixed $\theta$, then so does $F_{0} G\left(\left[e_{i}^{0}\right]\right)$. No matter what the choice of $F_{0}$, det $F_{0}>0$, the set $F_{0} G\left(\left\{e_{i}^{0}\right\}\right)$ is infinite. So $W(A, \theta)$ has an infinite number of potential wells, and there are $H^{k} \in G\left(\left\{e_{i}^{0}\right\}\right)$, $k=1,2,3, \ldots$ such that $\left|H^{k} F_{0}\right| \rightarrow \infty$. In fact, if $1+a \otimes n \in G\left(\left[e_{i}^{0}\right]\right)$, then $\mathrm{W}\left(\mathrm{A}\left(1+\lambda_{\mathrm{a}} \otimes \mathrm{n}\right)\right)$ is a periodic function of $\lambda$. If we associate $\mathrm{F}$ with the actual deformation of the body, it is clear that we will not be able to deform the body into one of these far away potential wells without compromising our notion of the lattice. That is, we expect dislocations to appear and to move with such large deformations, and they should surely contribute to the energy density.

Calculations of energy minima for an energy displaying the invariance $(2.4)$ and (2.5) by Chipot and Kinderlehrer [14] and Fonseca [31,32] support the inference above. However, they are useful for special loading devices, as we point out in the next section, and give some general insight into the nature of instabilities. At the present time, energy densities satisfying (2.4),(2.5) are the only ones for which the relaxed energy have been calculated.

Since dislocation movement appears unimportant for many transformation, it is plausible that the remarks above may be irrelevant. What is working remarkably well for many transformations is the assumption that $\mathrm{W}$ has the form (2.4) but that it has a domain $D$ restricted to include only a finite number of potential wells. In this framework, we choose $\left\{\mathrm{e}_{\mathrm{i}}^{\mathrm{o}}\right\}$ to be associated with the undistorted more symmetric phase and assume that 1 belongs to $D$. The objective is to delimit an appropriate neighborhood $D$ of 1 . A remarkably useful guide for this purpose has been nonlinear elasticity theory in its more classical form with invariance govemed by the subgroup $G\left(\left\{e_{i}^{o}\right\}\right) \cap S O(3)$, which is essentially a point group, in (2.4). A distillation of many recent calculations is this: choose $D$ to include the transformed lattice, i.e., to include a matrix $\mathrm{U}$ such that $L\left(\mathrm{Ue}_{1}^{\mathrm{o}}, \mathrm{Ue}_{2}^{\mathrm{o}}, \mathrm{Ue}_{3}^{\circ}\right)$ is the transformed lattice, require that $D$ be invariant under $\mathrm{G}\left(\left\{\mathrm{e}_{\mathrm{i}}^{\mathrm{o}}\right\}\right) \cap \mathrm{SO}(3)$ in the sense that $\mathrm{F} \in D \Leftrightarrow$ $F \in D\left[G\left(\left\{e_{i}^{0}\right]\right) \cap S O(3)\right]$, and finally require that $D$ not include any potential well minima not forced to be in it by the above criterion. Details of derivations of this type are given by Ball and James [7], Bhattacharya [11], Ericksen [30].

To understand what emerges of these considerations, let us consider a simple cubic to tetragonal transformation. 

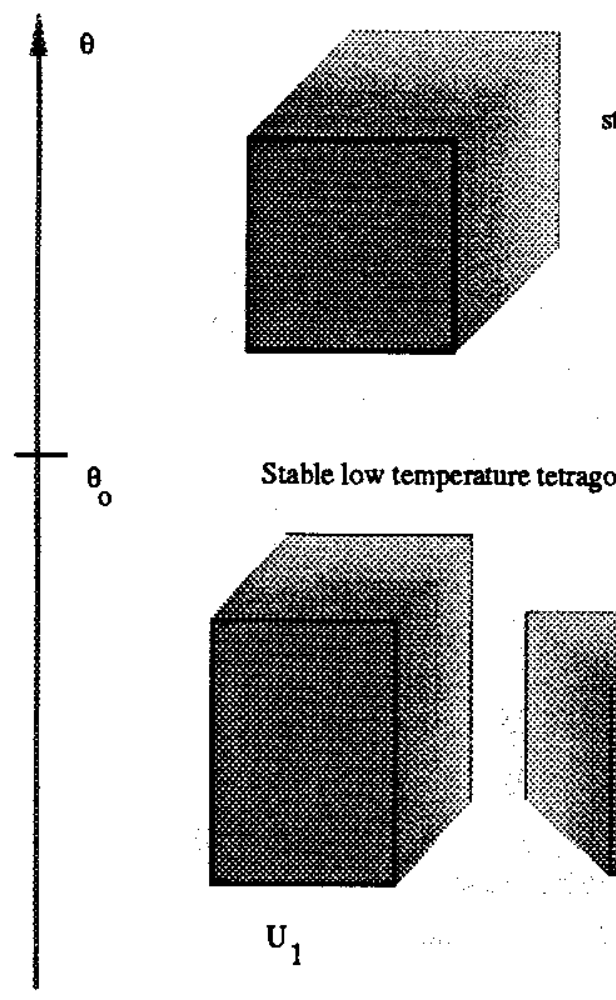

stable high temperature cubic phase

$F=\alpha 1$

\section{Stable low temperature tetragonal phase and variants}

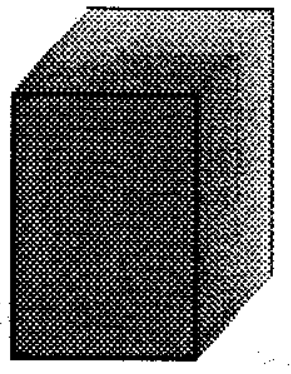

$\mathrm{U}_{1}$

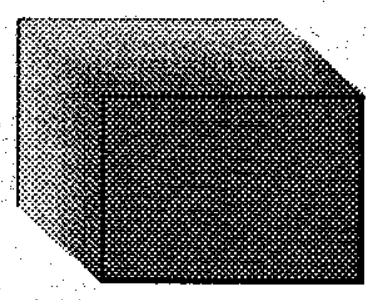

$\mathrm{U}_{2}$

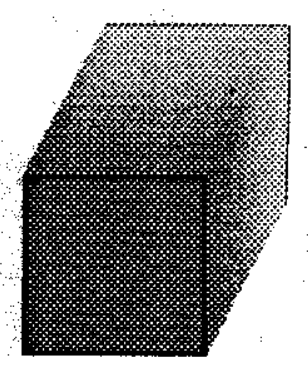

$\mathrm{U}_{3}$

Fig. 5 lllustration of a cubic/tetragonal transition

In this situation,

for $\theta>\theta_{0}$ : W(A, $\left.\theta\right)$ has strict minima on the orbit $\alpha(\theta) \mathrm{SO}(3)$ and for $\theta<\theta_{0}$ : W(A, $\quad$ ) has strict minima on the union of orbits $S O(3) U_{1}(\theta) \cup S O(3) U_{2}(\theta) \cup S O(3) U_{3}(\theta)$,

with $U_{i}(\theta)=\eta_{1}(\theta) 1+\left(\eta_{2}(\theta)-\eta_{1}(\theta)\right) e_{i}^{0} \otimes e_{i}^{0}$ (no sum), where the $\left\{e_{i}^{0}\right\}$ are orthonormal and the scalar valued functions $\alpha, \eta_{1}$, and $\eta_{2}$ are positive and continuous. Since $W$ is continuous, we necessarily have at the critical temperature

$$
\mathrm{W}\left(1, \theta_{0}\right)=\mathrm{W}\left(\mathrm{U}_{1}, \theta_{0}\right)=\mathrm{W}\left(\mathrm{U}_{2}, \theta_{0}\right)=\mathrm{W}\left(\mathrm{U}_{3}, \theta_{0}\right)
$$


Theory of diffusionless phase transitions

9

$1 / 10 / 89$

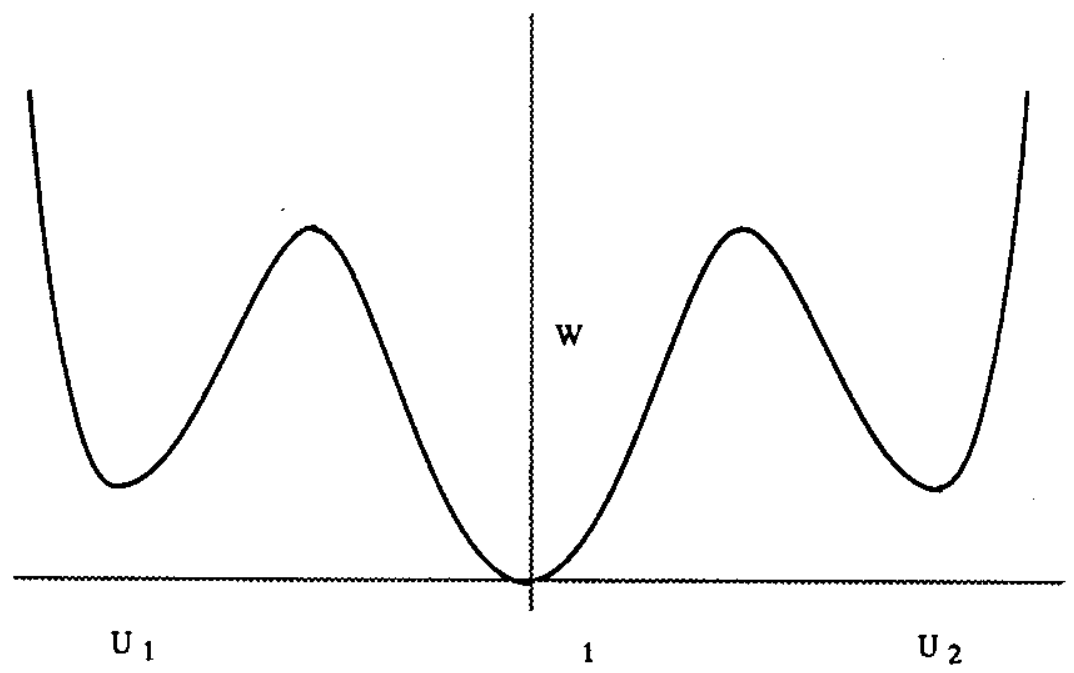

Fig. 6 Cubic phase at minimum energy above transition temperature.

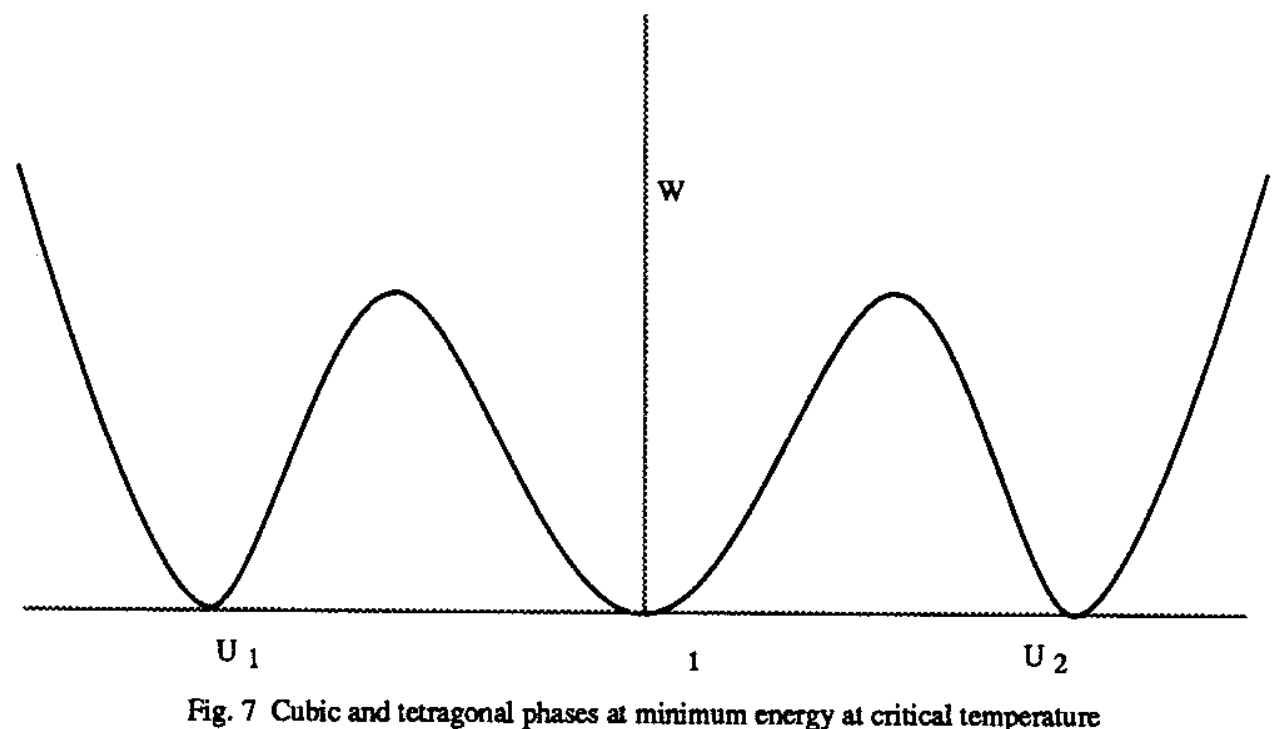

Fig. 7 Cubic and tetragonal phases at minimum energy at critical temperature 


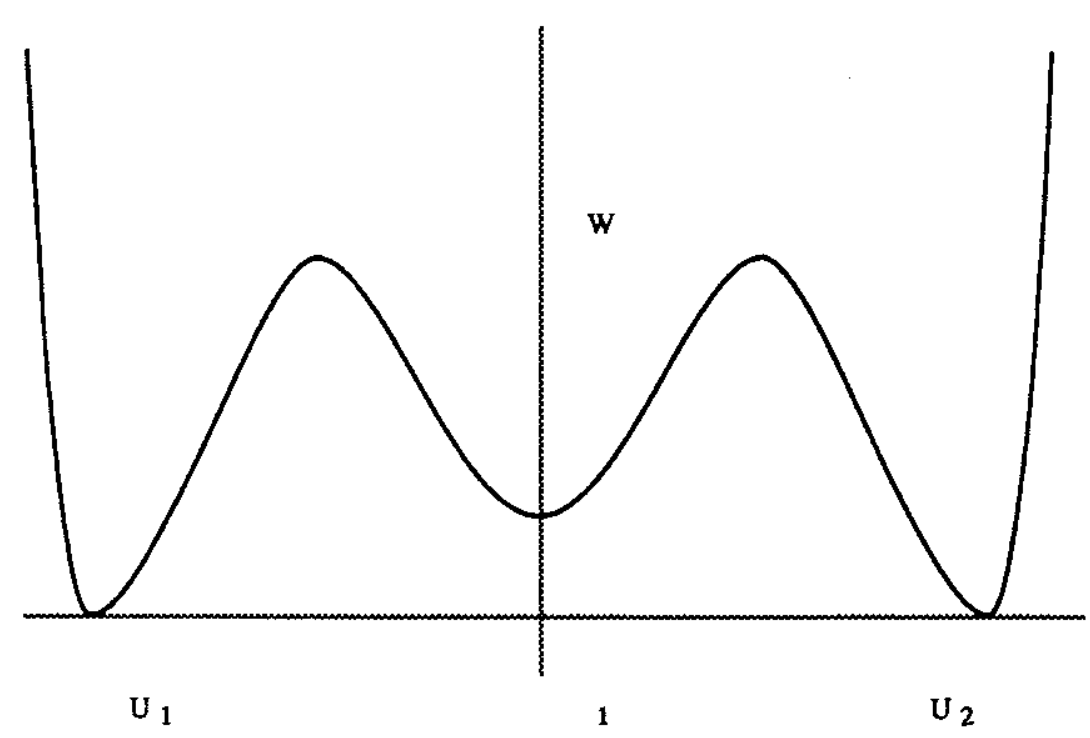

Fig. 8 Tetragonal phase at minimum energy below transition temperature

With these assumptions, as we shall describe in the ensuing sections, we may consider

$$
\text { inf } \int_{\Omega} \mathrm{W}(\nabla \mathrm{y}, \theta) \mathrm{dx}
$$

in a suitable class of functions $A$ for various values of $\theta$, and we are quickly led to consider the possibility that (2.7) has minimizers of the type (1.4), whatever that may mean. To describe these minimizers we frequently use the terminology

$$
\begin{aligned}
& \alpha(\theta) \mathrm{SO}(3) \quad \text { Austenite well, } \\
& \mathrm{SO}(3) \mathrm{U}_{1}(\theta) \cup \mathrm{SO}(3) \mathrm{U}_{2}(\theta) \cup \mathrm{SO}(3) \mathrm{U}_{3}(\theta) \quad \text { Martensite wells. }
\end{aligned}
$$

The typical situation for measured transformation strains is $\eta_{1} \neq 1$ and $\eta_{2} \neq 1$. In several known cases $\left(\eta_{1}\right)^{2} \eta_{2} \approx 1$. We assume that $\eta_{1} \neq 1 \neq \eta_{2}$. An easy calculation shows that there are no solutions of (1.4) with $\mathrm{F}$ and $\mathrm{A}$ on the same well. However we do find rank-one connections between any pair of martensite wells. The definite planes which can be planes of discontinuity, those of normal $\mathrm{n}$ in (1.4), arise from this calculation and provide an immediate first comparison between theory and experiment. In the cubic to tetragonal case, the planes are of the $(110)$ family and agree with those observed below the transformation temperature in many cubic to tetragonal transformations. 
However, there are no rank-one connections between austenite and martensite, whereas crystals at $\theta=\theta_{0}$ are observed to contain certain austenite/finely twinned martensite interfaces. These can be understood in terms of certain minimizing sequences similar to those constructed in $\S 1$. We describe them in $\$ 4$.

A notable feature of this development is that it contains no contribution for the surface energy which might be assigned to such surfaces of discontinuity. Thus some features of the microstructure which appear fine in the microscope are modelled as infinitely fine by the present theory. Typical interlaminar distances that occur in austenite/finely twinned martensite interfaces range from about $10 \mu \mathrm{m}$ down to a few atomic spacings, depending on the material. In a given material the fineness is approximately fixed. It is easy to ascribe such limited fineness to a small energy per unit area on the surfaces of gradient discontinuity, which would function to penalize the formation of arbitrarily fine laminates. Scaling calculations based on this idea do give reasonable qualitative results [6]. They predict, for example, that sufficiently small crystals will not contain an austenite/finely twinned martensite interface, an observed fact. A reasonable point of view is that in sufficiently large crystals, for example of diameter $>1 \mathrm{~mm}$, the surface energy serves just to pick out some fine scale, while the minimization of the bulk energy determines the kinematical properties of the microstructure on that scale. Furthermore, it is believed that the minimizing sequences of the bulk energy give reasonable values of the bulk properties like deformation, free energy, and stress, cf. $\$ 4$.

Of course, bulk theory alone will be useless for determining the scale of fineness or the precise details of the microstructure. It also will not give a complete description of growth phenomena. Recent work on this general problem is due to Gurtin [36]. Parry [55] and Fonseca [33] have discussed surface energy based on Herring's interpretation of the Wulff construction [37]. Recently, some thought has been given to the implications of minimizing the sum of bulk and surface energy [44].

Our lattice considerations here have been restricted to simple lattices where only mechanical interactions are presumed. Similar considerations are applicable to lattices with shifts or to polarizable dielectrics where $\mathrm{W}$ also depends on electrical polarization. In the latter case, the energy of a configuration as measured by (2.7) must include a potential which delivers the Maxwell stress. The theory for quartz is worked out in [40]. The paper [42] contains a discussion of ferroelectrics. 


\section{Principle of virtual work}

Common prejudice is to represent an equilibrium configuration at fixed temperature by a deformation $y(x)$ of a reference domain $\Omega$ which renders stationary the energy functional; that is,

$$
\delta \int W(\nabla y, \theta) d x=0
$$

In the present situation, it is not easy to justify this since any affine $y(x)$ satisfies (3.1) and we have seen that many are unstable. We prefer to think in terms of stability criteria, but, unfortunately, we are lacking guidelines in this regard. About these issues, much has been written, $[2,3,9,14,57,58]$. To surmount these difficulties, at least from the analytical point of view, we shall resort to direct methods.

Provisionally, this issue may be separated into two parts. The first is to seek the minimum energy available to a configuration, which is the topic of this section. Second, we may ask about the deformation which delivers this minimum, the values of various state functions, and the determination of fine scale or microstructural features. Recall that the two parts are separate because the functional of (3.1) is not lower semicontinuous. Hence, as illustrated in the previous sections, the loading may give rise to a macroscopic deformation which only represents a local spatial average over potential wells, whose energy need not be a minimum.

Given a domain $\Omega \subset \mathbb{R}^{3}$ with suitably smooth boundary and $y_{0} \in H^{1, \infty}(\Omega)$ with $\operatorname{det} \nabla y_{0}>0$, let

$$
A=A_{\Omega}\left(\mathrm{y}_{0}\right)=\left\{\mathrm{u} \in \mathrm{H}^{1, \infty}(\Omega): \operatorname{det} \nabla \mathrm{u}>0 \text { and } \mathrm{u}=\mathrm{y}_{0} \text { on } \partial \Omega\right\}
$$

and set

$$
E_{\Omega}\left(\mathrm{y}_{0}, \theta\right)=\inf _{A} \int_{\Omega} \mathrm{W}(\nabla \mathrm{u}, \theta) \mathrm{dx}
$$

Here we briefly discuss the evaluation of (3.3). An analysis undertaken in this spirit is sometimes called "relaxation," and one objective is to replace, at least temporarily, the original density $\mathrm{W}$ by a density $W^{*}$ which is lower semicontinuous and for which 


$$
E_{\Omega}\left(\mathrm{y}_{0}, \theta\right)=\inf _{A} \int_{\Omega} W^{\#}(\nabla \mathrm{u}, \theta) \mathrm{dx} .
$$

Necessarily, if (3.3) holds, then in particular whenever $y_{0}(x)=F_{0} x$ is affine, det $F_{0}>0$, we have that

$$
\begin{aligned}
& \mathrm{W}^{\#}\left(\mathrm{~F}_{\mathrm{o}}, \theta\right)|\Omega|=\inf _{H} \int_{\Omega} \mathrm{W}\left(\mathrm{F}_{\mathrm{o}}+\nabla \zeta, \theta\right) \mathrm{dx}, \\
& H=\left\{\zeta \in \mathrm{H}_{\mathrm{o}}^{1, \infty}(\Omega) ; \operatorname{det}\left(\mathrm{F}_{\mathrm{o}}+\nabla \zeta\right)>0\right\} .
\end{aligned}
$$

The proof of (3.4) follows from an easy adaptation of the argument of [6] . Were the kinematical constraint on the competing functions absent, it would be possible to show that (3.3) is verified for any data $y_{0} \in H^{1, \infty}(\Omega)$, but we are only able to establish this in limited, albeit useful, circumstances. On a deeper mechanical level, it is very difficult to actually know the function $W^{\#}$ in terms of $\mathrm{W}$.

In his discussion of the Gibbs phenomenon for thermoelastic solids, Ericksen [25] observed that the energy of a homogeneously deformed body in equilibrium with a heat bath might tend to assume the value of a certain subenergy which he defined as

$$
\varphi(\operatorname{det} F, \theta)=\inf _{\operatorname{det} A} A \operatorname{det} F W(A, \theta)
$$

Ericksen illustrated that this was consistent with certain types of thermodynamical thought where critical temperatures state functions were computed or measured in terms of specific volume alone, ignoring other features of the deformation.

We are able to give an additional interpretation to this subenergy for a crystalline solid when (2.4) and (2.5), namely, invariance under the full lattice group, is assumed. Here it is possible to show that

$$
W^{*}(F, \theta)=\varphi^{* *}(\operatorname{det} F, \theta), \quad \operatorname{det} F>0,
$$

where $\varphi^{* *}(t, \theta)$ is the convexification of the function $\varphi(t, \theta)$ as a function of the real variable $t$. For the proof of this we refer to $[14,32]$. In this case, $W^{\#}$ is polyconvex, namely, a convex function of $\mathrm{A}, \operatorname{adj} \mathrm{A}$, and det $\mathrm{A}$, but for more restricted invariance groups, we do not expect this. 
Two examples where (3.3) holds may be worthwhile mentioning.

A. Assume that $\mathrm{W}$ satisfies (2.4) and (2.5) so that $\mathrm{W}^{\#}$ is given by (3.6). If $\mathrm{y}_{\mathrm{o}} \in \mathrm{Cl}^{1}(\bar{\Omega})$ satisfies

$$
\operatorname{det} \nabla \mathrm{y}_{0}=\mathrm{v} . \quad \text { in } \Omega \text {, }
$$

where $v$ is constant, then

$$
E_{\Omega}\left(\mathrm{y}_{0}, \theta\right)=\inf _{A} \int_{\Omega} \varphi^{* *}(\operatorname{det} \nabla \mathbf{u}, \theta) \mathrm{dx}=\varphi^{* *}(\mathrm{u}, \theta)|\Omega|
$$

B. Assume that inf $\mathrm{W}=0$ and that $E_{\Omega}\left(\mathrm{y}_{0}, \theta\right)=0$. Then

$$
E_{\Omega}\left(\mathrm{y}_{0}, \theta\right)=\inf _{A} \int_{\Omega} \mathrm{W}^{\#}(\nabla \mathrm{u}, \theta) \mathrm{dx}=0
$$

Although it appears a banality, a consequence of part B is that it assists us to calculate all possible deformation gradients $F$ for which $W^{\#}(F, \theta)=0$ when $W$ is assumed to have a given well structure.

There is an extensive literature about relaxation and lower semicontinuity of variational integrals, $[1,2,13,18,19,45-49,59,66]$.

4 Young measures: state functions, and microstructure

We now arrive at the place where we wish to relate the minimum energy of a configuration with the deformation which arises as the limit of a minimizing sequence. As we have suggested earlier, the technical device we adopt for this purpose is the Young measure, or parametrized measure. We shall give to it an interpretation in terms of local spatial averages and illustrate how it may be employed to analyze properties of equilibrium configurations. The use of Young measures in differential equations was first introduced by Tartar [68-70], especially in order to study hyperbolic conservation laws. It will serve us as an accounting tool to summarize the oscillatory properties of a minimizing sequence. 
Let us assume that we are in the favorable situation where (3.3) holds, so the infimum of energy is the same as the infimum of the relaxed energy. Further, suppose we have in hand a sequence $\mathrm{y}^{\mathbf{k}} \in A_{\Omega}\left(\mathrm{y}_{0}\right)$ such that

$$
\begin{aligned}
E_{\Omega}\left(\mathrm{y}_{0}, \theta\right) & =\lim _{\mathbf{k} \rightarrow \infty} \int_{\Omega} W^{\#}\left(F^{\mathbf{k}}, \theta\right) d x=\lim _{\mathbf{k} \rightarrow \infty} \int_{\Omega} W\left(F^{\mathbf{k}}, \theta\right) d x, \\
F^{k} & =\nabla y^{k},
\end{aligned}
$$

and moreover

$$
y^{k} \rightarrow y \quad \text { in } H^{1, \infty}(\Omega) \text { weak*. }
$$

By choice of a subsequence, if necessary, this will be the case provided $\left\|F^{k}\right\|_{L^{\infty}(\Omega)} \leq C<\infty$ for some constant $\mathrm{C}$.

First let us remind ourselves that (4.2) means

$$
\begin{aligned}
& \mathrm{y}^{\mathrm{k}} \rightarrow \mathrm{y} \text { uniformly in } \bar{\Omega} \text { and } \\
& \int_{\mathrm{E}} \mathrm{F}^{\mathrm{k}} \mathrm{dx} \rightarrow \int_{\mathrm{E}} \mathrm{Fdx} \quad \text { for all (measurable) } \mathrm{E} \subset \Omega,
\end{aligned}
$$

where $F=\nabla y$. Pointwise convergence of the sequence ( $F^{k}$ ) will ordinarily fail.

A general fact is that given a sequence $\left(f^{k}\right)$ which is bounded in $L^{\infty}(\Omega)$, it has a subsequence ( $f^{\prime}$ ) and an $f \in L^{\infty}(\Omega)$ such that

$$
\mathrm{fk}^{\prime} \rightarrow \mathrm{f} \quad \text { in } \mathrm{L}^{\infty}(\Omega) \text { weak*, }
$$

namely, (4.3) 2 . So given a continuous function $\psi(A)$ defined on $3 \times 3$ matrices, the sequence ( $\psi\left(F^{k}\right)$ ) is bounded in $L^{\infty}(\Omega)$ and thus for some subsequence, which we continue to denote by ( $\psi\left(F^{k}\right)$ ), there is a $\bar{\psi} \in \mathrm{L}^{\infty}(\Omega)$ for which

$$
\Psi\left(F^{h}\right) \rightarrow \bar{\psi} \quad \text { in } L^{\infty}(\Omega) \text { weak* }
$$


Inasmuch as usually the function $\bar{\psi}$ is not the same as $\psi(\mathrm{F})$, what is it? This is the question to which the Young measure responds. In the present framework, there is a family $\left(v_{x}\right)_{x \in \Omega}$ of probabability measures defined on the set $M$ of matrices, and depending measurably on $x \in \Omega$, such that

$$
\bar{\psi}(\mathrm{x})=\int_{\mathbf{M}} \psi(\mathrm{A}) \mathrm{d} v_{\mathbf{x}}(\mathrm{A}) \text { a.e. in } \Omega
$$

Since $F^{k} \rightarrow F$ in $L^{\infty}(\Omega)$ weak*, we have immediately that

$$
F(x)=\int_{\mathbf{M}} A d v_{x}(A) \quad \text { a.e. in } \Omega \text {. }
$$

In the simple but important case of (1.6), one easily verifies that

$$
v_{\mathrm{x}}=(1-\lambda) \delta_{\mathrm{F}}+\lambda \delta_{\mathrm{A}}
$$

The oscillatory nature of the sequence $\left(u^{k}\right)$ is recorded in the variant proportion $\lambda$. In fact, it is obvious that given $x_{0} \in \Omega$,

$$
\left.1-\lambda=\lim _{\rho \rightarrow \infty} \lim _{k \rightarrow \infty} \frac{1}{\left|B_{p}\right|} \mid x \in B_{p}\left(x_{0}\right): \nabla u^{k}(x)=F\right\} \mid .
$$

The analysis of the Young measure $\left(v_{x}\right)_{x \in \Omega}$ rests upon understanding the role of weak* continuous and lower semicontinuous functions $\psi(A)$. In general, these are the minors of the matrix $A$, explicitly, the nineteen functions $A$, adj $A=\operatorname{det} A A^{-T}$, and $\operatorname{det} A$. This means that

$$
\operatorname{adj} F^{k} \rightarrow \operatorname{adj} F \quad \text { and } \quad \operatorname{det} F^{k} \rightarrow \operatorname{det} F \quad \text { in } L^{\infty}(\Omega) \text { weak*. }
$$

Employing our notation (4.5), we may write

$$
\begin{aligned}
& \operatorname{adj} F(x)=\int_{M} \operatorname{adj} A d v_{x}(A) \text { and } \\
& \operatorname{det} F(x)=\int_{M} \operatorname{det} A d v_{x}(A) \\
& \text { a.e. in } \Omega \text {. }
\end{aligned}
$$


Now $\mathrm{W}^{\#}$ is lower semicontinuous, so we obtain from (4.1) and our hypothesis

(3.3) that

$$
\begin{aligned}
\int_{\Omega} W^{\#}(F, \theta) d x \leq \lim _{k} \rightarrow \infty & \int_{\Omega} W^{\#}\left(F^{k}, \theta\right) d x \\
& =E_{\Omega}\left(y_{0}, \theta\right) \leq \int_{\Omega} W^{\#}(F, \theta) d x
\end{aligned}
$$

so that

$$
\int_{\Omega} W^{\#}(F, \theta) d x=\lim _{\mathbf{k} \rightarrow \infty} \int_{\Omega} W^{\#}\left(F^{k}, \theta\right) d x=\lim _{\mathbf{k}} \rightarrow \infty \int_{\Omega} W\left(F^{k}, \theta\right) d x .
$$

A consequence of this is that the sequences $\left(W^{\#}\left(F^{k}, \theta\right)\right)$ and $\left(W\left(F^{k}, \theta\right)\right)$ both converge weak* to $\mathrm{W}^{\#}(\mathrm{~F}, \theta)$, so we discover a twentieth weak* continuous function, one particular to the minimizing sequence. Again employing our notation (4.5),

$$
W^{\#}(F(x), \theta)=\int_{M} W^{\#}(A, \theta) d v_{\mathbf{x}}(A)=\int_{M} W(A, \theta) d v_{\mathbf{x}}(A) \quad \text { a.e. in } \Omega
$$

Since $\mathrm{W}^{\#} \leq \mathrm{W}$, we deduce that

$$
\operatorname{supp} v_{\mathbf{x}} \subset\left\{A \in \mathbb{M}: W^{\#}(A, \theta)=W(A, \theta)\right\}
$$

which is our first statement about the Young measure associated to the minimizing sequence ( $y^{k}$ ). It will tum out that the algebraic structure of the set on the right in (4.10) may then limit or determine possible configurations and their microstructure. For example, in the austenite/martensite transition described in $\$ 2$, the Young measure $\left(v_{x}\right)_{x \in \Omega}$ corresponding to any energy minimizing configuration at critical temperature $\theta_{0}$ will have

$$
\text { supp } v_{\mathbf{x}} \subset \text { austenite well } \cup \text { martensite weils . }
$$

Special choices of $\psi$ in (4.5) provide us with the state functions of the configuration. For example, set $S(A, \theta)=\partial W(A, \theta) / \partial A^{\prime}$, the Piola - Kirchhoff stress. We might write that 


$$
\bar{S}(x, \theta)=\int_{M} S(A, \theta) d v_{x}(A) \quad \text { in } \Omega
$$

This has a variational justification if $\operatorname{det} F(x) \geq c>0$ in $\Omega$. In this case,

$$
\begin{array}{r}
\left.\frac{d}{d \varepsilon} \int_{\Omega} \int_{M} W(A+\varepsilon \nabla \zeta) d v_{x}(A) d x\right|_{\varepsilon=0}=\iint_{\Omega M} S(A) \cdot \nabla \zeta d v_{x}(A) d x \\
\text { for } \zeta \in H_{0}^{1, \infty}(\Omega) .
\end{array}
$$

Then $W-W^{\#}$ achieves its minimium value on $\operatorname{supp} v_{\mathbf{x}}$, so if $W^{\#}$ is differentiable, with $S^{\#}(A)$ $=\partial \mathrm{W}^{\#}(\mathrm{~A}, \theta) / \partial \mathrm{A}$, it follows from $(4.9)$ that

$$
\bar{S}(x, \theta)=\bar{S}^{\#}(x, \theta)=S^{\#}(F(x)) \text { a.e. in } \Omega \text {, }
$$

and this expression may be substituted into (4.12). Thus the state of stress in the body may always be described by a "relaxed" field equation,

$$
-\operatorname{div} S^{\#}(F(x))=0 \text { a.e. in } \Omega \text {. }
$$

Of course, the second derivatives of $W$ with respect to $A$ do not agree with the second derivatives of $W^{\#}$. One discussion of linearization in this context and its relationship to homogenization is given in [15].

A second example of a state function easily computable is the specific heat at constant volume,

$$
C_{v}=-\frac{1}{\theta} \frac{\partial^{2} W}{\partial \theta^{2}}
$$

Again in this case, since $\bar{W}(x, \theta)=W^{\#}(F(x), \theta)$,

$$
C_{v}=-\frac{1}{\theta} \frac{\partial^{2} W^{\#}(F(x), \theta)}{\partial \theta^{2}}=-\frac{1}{\theta} \int_{M} \frac{\partial^{2} W}{\partial \theta^{2}}(A, \theta) d v_{x}(A)
$$

In the special case of the symmetry of $(2.4)$ and $(2.5)$, where $W^{\#}(F, \theta)=\varphi^{* *}(\operatorname{det} F, \theta)$, 


$$
C_{v}=-\frac{1}{\theta} \frac{\partial^{2} \varphi^{* *}(\operatorname{det} F(x), \theta)}{\partial \theta^{2}}
$$

which depends only on the specific volume. In particular, the points where the right hand side is not differentiable, which are usually the transition temperatures like $\theta_{0}$ in $(2.6)$, are determined by the specific volume alone. This is consistent with some views of the matter and some experimental practice, cf. eg. Pippard [60].

The examples above may be termed "macroscopic" in the sense that the results do not depend on the particular Young measure $\left(v_{x}\right)_{x} \in \Omega$. Let us now briefly discuss some "microstructural" properties, which do depend on the particular Young measure. This will also introduce some curious analytical questions as well.

There are, in essence, two equivalent ways in which the Young measure may be considered a local spatial average, Ball [5,\$4] and [14,Theorem 7.3]. Given $a \in \Omega$ and $p>0$, define the measure $v_{a, \rho}^{k}$ by

$$
\left\langle v_{a, p}^{k}, \Psi\right\rangle=\frac{1}{B_{\rho}} \int_{B_{p}(a)} \psi\left(F^{k}(x)\right) d x
$$

This defines a continous linear functional on $C(K)$ for $K=\{A:|A| \leq C\}$, cf. (4.2) et. seq. This measure is a probability measure giving the distribution of values of $F^{k}(x)$ in $B_{\rho}(a)$. By the weak convergence of the sequence $\left(\mathrm{F}^{\mathrm{k}}\right)$,

$$
\begin{aligned}
& v_{\mathrm{a}, \rho}^{\mathrm{k}} \rightarrow v_{\mathrm{a}, \rho} \quad \quad \text { weak* as measures as } \mathbf{k} \rightarrow \infty \text {, where } \\
& \left\langle v_{\mathrm{a}, \rho}, \Psi\right\rangle=\frac{1}{\left|\mathrm{~B}_{\mathrm{p}}\right|} \int_{\mathrm{B}_{\rho}(\mathrm{a})} \int_{\mathrm{M}} \Psi(\mathrm{A}) \mathrm{d} v_{\mathrm{x}}(\mathrm{A}) \mathrm{dx} .
\end{aligned}
$$

By Lebesgue's Theorem,

$$
\left\langle v_{a}, \rho, \psi\right\rangle \rightarrow\left\langle v_{a}, \Psi\right\rangle \quad \text { as } \rho \rightarrow 0 \text { a.e. in } \Omega,
$$

from which it follows by the separability of $\mathrm{C}(\mathrm{K})$ that 


$$
v_{\mathrm{a}, \rho} \rightarrow v_{\mathrm{a}} \quad \text { weak }^{*} \text { as measures, a.e. in } \boldsymbol{\Omega} \text {. }
$$

These obvious remarks have the consequence that we may restrict $v_{a}$ for almost every $a \in \Omega$. In other words, by choosing a diagonal sequence of $(k, \rho)$, one verifies that the family of measures

$$
\left(\mu_{x}\right)_{x \in \Omega} \text {, where } \mu_{x}=v_{a} \text { for all } x \in \Omega \text {, }
$$

is also a parametrized measure which is determined by a sequence of deformation gradients.

Another view of this is given by Matos [50].

The sequence $\left(v_{a, p}\right)$ admits a convergent subsequence at every $a \in \Omega$, even if this is not an obvious spatial average. This will be useful in what follows.

We turn now to a few specific examples of the analysis of Young measures. As our first example, we shall show that a Young measure $\left(v_{x}\right)_{x \in \Omega}$ supported on a single well $\operatorname{SO}(3) \mathrm{F}_{0}$ for each $x \in \Omega$ reduces to a constant Dirac mass.

We may assume that $F_{0}=1$ for the argument that follows by a change of variables.

Suppose that $F(x)=\nabla y(x)$ is the underlying deformation in the sense of (4.6) as usual. Then

$$
\begin{aligned}
& F(x)=\int_{S O(3)} Q d v_{x}(Q) \text { and } \\
& \operatorname{adj} F(x)=\int_{S O(3)} Q d v_{x}(Q)=F(x),
\end{aligned}
$$

since $\operatorname{adj} Q=Q$ when $Q$ is a proper rotation. Finally,

$$
\operatorname{det} F(x)=\int_{S O(3)} d v_{x}(Q)=1
$$

Thus

$$
F(x)=\operatorname{det} F(x) F(x)^{-T}=F(x)^{-T},
$$


so $F(x)$ is a rotation. Now $S O(3) \subset$ sphere of radius $\sqrt{3}$ in $\mathbb{R}^{9}$, which is strictly convex, so the only way (4.20) can hold is if

$$
v_{\mathbf{x}}=\delta_{\mathrm{F}(\mathrm{x})} \text { a.e. in } \Omega \text {. }
$$

A more efficient and general proof of this is given in Ball [4].

The second part of the argument is due essentially to Reshetnyak $[64,65]$. Of course we know that any smooth deformation gradient $F(x)$ satisfying $F(x) \in S O(3)$ must be constant. Let

$$
U(A)=\frac{1}{2}|A|^{2}-\operatorname{det} A \text {, }
$$

which has $U_{A}(A)=A-\operatorname{det} A$. Since $\operatorname{det} A$ is a null-lagrangian, the Euler equation of $U$ is

$$
-\operatorname{div} U_{A}(\nabla u)=\Delta u
$$

If $Q$ is any rotation, $\operatorname{det} Q=1$, then $U_{A}(Q)=0$, so for the $F(x)$ of $(4.20), U_{A}(F(x))=0$; whence,

$$
\Delta y=-\operatorname{div} U_{A}(\nabla y)=0 \text { in } \Omega
$$

in the sense of $H^{1}$, say. Hence $y$ is smooth and by the remark above about smooth deformations, $\nabla y(x)=F(x)=R$, for some rotation $R$, and

$$
v_{\mathrm{x}}=\delta_{\mathrm{R}} \quad \text { a.e. in } \Omega \text {. }
$$

This conclusion agrees with our abundant observations that pure austenite or a pure variant of martensite cannot form a microstructure. It is a sort of Young measure regularity theorem: knowledge of the support of the measure at each point determines its global behavior. 


\section{Young Measures: the Austenite /Martensite Transition}

As a second example, consider the most common microstructure observed in a thermoelastic martensitic alloy at the transformation temperature, the austenite/finely twinned martensite interface, shown schematically in Fig. 9. As explained in \$2, there are no rank-one connections between the austenite well and any of the martensite wells, so we cannot understand this microstructure in terms of piecewise differentiable functions. Moreover, the observation that the martensite variants are always observed to be finely twinned suggests the study of minimizing sequences. Our analysis is in the style of [6].

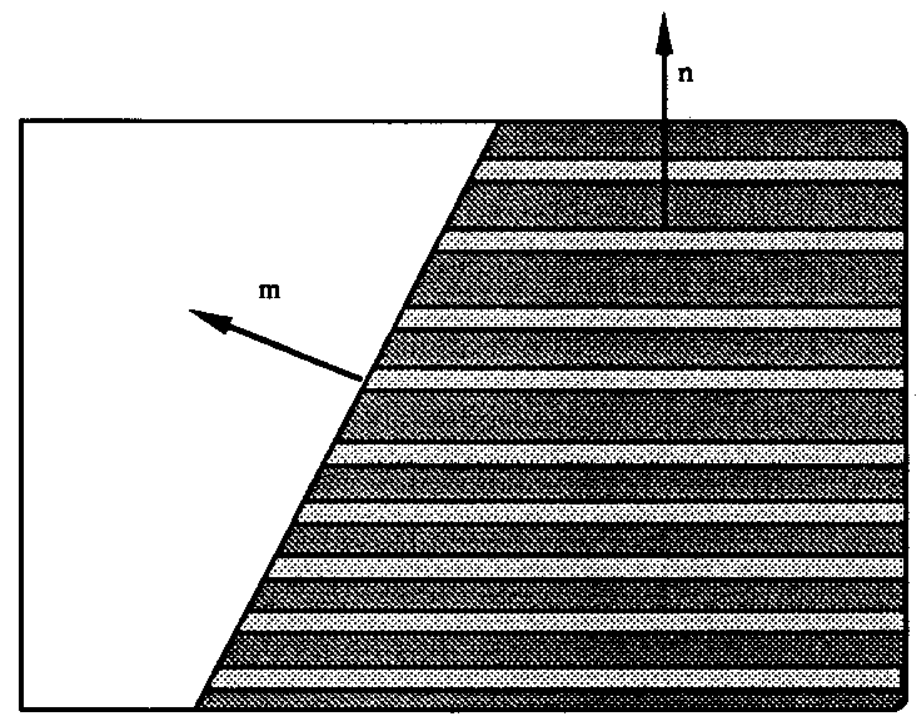

Fig. 9 Austenite/finely twinned martensite interface showing variants of martensite and the kinematical quantities $m$ and $n$.

Observation of the photomicrographs quickly convinces one of a salient feature: there are essentially three deformation gradients involved, two of which belong to martensite wells and which differ by a rank-one matrix and one which belongs to the austenite well. Being noncomittal about the precise geometry of the microstructure, we therefore assume simply that we have in hand a sequence $y^{k} \rightarrow y$ in $H^{1, \infty}$ which is a minimizing sequence for the problem (2.7) and that its Young measure $\left(v_{x}\right)_{x \in \Omega}$, according to remark $B$ of $\S 3$, satisfies 


$$
\begin{gathered}
\operatorname{supp} v_{x} \subset\left\{M_{1}, M_{2}, C\right\} \quad \text { a.e. in } \Omega \text {, where } \\
M_{2}-M_{1}=a \otimes n, C-M_{1} \neq \text { rank one, and } C-M_{2} \neq \text { rank one, }
\end{gathered}
$$

for vectors a, $n \in \mathbb{R}^{3}$. Of course we have in mind that $\mathrm{M}_{i}=R_{i} U_{i}, i=1,2$, and $C=R$, where $R_{1}, R_{2}$, and $R \in S O(3)$, and $U_{i}$ are from (2.6).

Our interest lies in the observation that the presence of a microstructure involving the austenite and the martensite imposes restrictions on the matrices $M_{1}, M_{2}$, and $C$. We shall show that for some $\sigma, 0<\sigma<1$,

$$
\operatorname{rank}\left[C-\left((1-\sigma) M_{1}+\sigma M_{2}\right)\right]=1 \text {, }
$$

that is, the two martensite variants are capable of averaging among themselves so that the result is kinematically compatible with the austenite. In addition, we illustrate how the presence of an austenite/martensite interface determines how the microstructure propagates in the sample. We shall show that there is a "propagation region", cf. (5.28), consisting of a mixture of austenite and finely twinned martensite which occurs with the fixed variant proportions $1-\sigma$ and $\sigma$. This is summarized by the reperesentation of the Young measure given in (5.29).

For simplicity, we shall assume that the reference configuration $\Omega$ is convex. Given the sequence $\left(y^{k}\right)$, let $\left(v_{x}\right)_{x \in \Omega}$ denote the Young measure. By (5.1),

$$
v_{x}=\lambda_{1}(x) \delta_{M_{1}}+\lambda_{2}(x) \delta_{M_{2}}+\lambda_{3}(x) \delta_{C}, \quad \sum \lambda_{i}(x)=1 \text { a.e. in } \Omega
$$

Step 1 If $\left\{0<\lambda_{3}<1\right\}$ has positive measure, then (5.3) holds.

In this case, for almost every $a \in\left(0<\lambda_{3}<1\right\}$, we may consider the restricted Young measure $v_{a}$. Let us set $\mu=v_{a}$. Since a may be chosen a Lebesgue point of $\lambda_{i}$, we may write

$$
\mu=\tau_{1} \delta_{M_{1}}+\tau_{2} \delta_{M_{2}}+\tau_{3} \delta_{C}, \quad \sum \tau_{i}=1, \quad 0<\tau_{3}<1
$$

Let $F$ denote the underlying deformation of $\mu$. Again we have the relations for the minors of $F$, which are

$$
F=\tau_{1} M_{1}+\tau_{2} M_{2}+\tau_{3} C
$$




$$
\begin{aligned}
& \operatorname{adj} F=\tau_{1} \operatorname{adj} M_{1}+\tau_{2} \operatorname{adj} M_{2}+\tau_{3} \operatorname{adj} C, \text { and } \\
& \operatorname{det} F=\tau_{1} \operatorname{det} M_{1}+\tau_{2} \operatorname{det} M_{2}+\tau_{3} \operatorname{det} C .
\end{aligned}
$$

We now consider the identity

$$
\begin{aligned}
\operatorname{adj}\left(\sum_{1}^{3} \alpha_{i} A_{i}\right)= & \sum_{1}^{3} \alpha_{i} \operatorname{adj} A_{i}-\frac{\alpha_{1} \alpha_{2}}{1-\alpha_{3}} \operatorname{adj}\left(A_{2}-A_{1}\right)- \\
& \alpha_{3}\left(1-\alpha_{3}\right) \operatorname{adj}\left[\left(\frac{\alpha_{1}}{1-\alpha_{3}} A_{1}+\frac{\alpha_{2}}{1-\alpha_{3}} A_{2}\right)-A_{3}\right],
\end{aligned}
$$

which holds for any scalars $\alpha_{i}$ with $\alpha_{1}+\alpha_{2}+\alpha_{3}=1, \alpha_{3} \neq 0,1$, and $3 \times 3$ matrices $A_{i}$. Applying this to (5.6) 1 and comparing it with $(5.6)_{2}$, using that $\operatorname{adj}\left(M_{2}-M_{1}\right)=0$, gives

$$
\operatorname{adj}\left[\frac{\tau_{1}}{1-\tau_{3}} M_{1}+\frac{\tau_{2}}{1-\tau_{3}} M_{2}-C\right]=0
$$

Hence,

$$
C-\left[(1-\sigma) M_{1}+\sigma M_{2}\right]=b \otimes m,
$$

for some $b, m \in \mathbb{R}^{3}$ with $\sigma=\frac{\tau_{2}}{1-\tau_{3}} \in(0,1)$. Since $b \otimes m$ is not zero, by $(5.6) 2,3$, the result (5.3) follows.

In the rest of the discussion, we shall assume that $\lambda_{3}=0$ or 1 . So we may write

$$
\lambda_{3}=\chi \Omega-\mathrm{E} \text { and } \lambda_{1}+\lambda_{2}=\chi \mathrm{E}
$$

where $\mathrm{E}$ is the "martensite" set and $\Omega-\mathrm{E}$ is the "austenite" set with respect to the reference configuration. We assume that $\mathrm{E}$ is not of full measure. The major issue here will be to show that (5.3) holds.

Step 2 The basic relation

From (5.4) and (5.8), 


$$
F(x)=\nabla y(x)=\chi E\left(\lambda_{1} M_{1}+\lambda_{2} M_{2}\right)+\left(1-\chi_{E}\right) C \quad \text { in } \Omega
$$

so

$$
\mathrm{F}(\mathrm{x})-\mathrm{C}=\chi \mathrm{E}\left(\lambda_{1}\left(\mathrm{M}_{1}-\mathrm{C}\right)+\lambda_{2}\left(\mathrm{M}_{2}-\mathrm{C}\right)\right) \quad \text { in } \Omega
$$

Introduce

$$
z(x)=y(x)-C x \text { and } N_{i}=M_{i}-C, i=1,2
$$

With these notations,

$$
\begin{aligned}
& \nabla z=\chi \mathrm{E}\left(\lambda_{1} \mathrm{~N}_{1}+\lambda_{2} \mathrm{~N}_{2}\right) \quad \text { in } \Omega \text {, where } \\
& \lambda_{1}+\lambda_{2}=1 \text { in } \mathrm{E} \text { and } \mathrm{N}_{2}=\mathrm{N}_{1}+\mathrm{a} \otimes \mathrm{n} .
\end{aligned}
$$

By the hypothesis (5.2), $\operatorname{rank} \mathrm{N}_{\mathrm{i}} \geq 2$.

Observe that since, by the equality of mixed second partials,

$$
\int_{\Omega}\left(\frac{\partial z^{i}}{\partial x_{\alpha}} \frac{\partial \zeta}{\partial x_{\beta}}-\frac{\partial z^{i}}{\partial x_{\beta}} \frac{\partial \zeta}{\partial x_{\alpha}}\right) d x=0,
$$

we have that

$\mathrm{N}_{1 \alpha}^{\mathrm{i}} \int_{\Omega} \lambda_{1} \frac{\partial \zeta}{\partial \mathrm{x}_{\beta}} \mathrm{dx}+\mathrm{N}_{2 \alpha}^{\mathrm{i}} \int_{\Omega} \lambda_{2} \frac{\partial \zeta}{\partial \mathrm{x}_{\beta}} \mathrm{dx}=\mathrm{N}_{1 \beta}^{\mathrm{i}} \int_{\Omega} \lambda_{1} \frac{\partial \zeta}{\partial \mathrm{x}_{\alpha}} \mathrm{dx}+\mathrm{N}_{2 \beta}^{\mathrm{i}} \int_{\Omega} \lambda_{2} \frac{\partial \zeta}{\partial \mathrm{x}_{\alpha}} \mathrm{dx}$

Let us set

$$
\xi=\int_{\Omega} \lambda_{1} \nabla \zeta \mathrm{dx} \text { and } \eta=\int \lambda_{2} \nabla \zeta \mathrm{dx}=\int_{\Omega}\left(\chi \mathrm{E}-\lambda_{1}\right) \nabla \zeta \mathrm{dx}
$$

so (5.12) becomes our basic relation:

$$
N_{1 \alpha}^{i} \xi_{\beta}+N_{2 \alpha}^{i} \eta_{\beta}=N_{1 \beta}^{i} \xi_{\alpha}+N_{2 \beta}^{i} \eta_{\alpha}
$$

or, 


$$
(c \cdot \xi) N_{1}+(c \cdot \eta) N_{2}=N_{1} c \otimes \xi+N_{2} c \otimes \eta \text {, for any } c \in \mathbb{R}^{3} \text {. }
$$

Note that if $\xi=0$, then

$$
c \cdot \eta N_{2}=N_{2} c \otimes \eta
$$

so $\mathrm{N}_{2}$ is of rank one, unless $\eta=0$. This is a contradiction. Similarly, $\eta=0$ implies that $\xi$ $=0$. We conclude that

$$
\xi=0 \Leftrightarrow \eta=0 \text { and } \xi+\eta \neq 0 \text { for some } \zeta \in C_{0}^{\infty}(\Omega) \text {. }
$$

The last assertion follows from the assumption that $E$ is not of full measure.

Step 3 There are vectors $b, m \in \mathbb{R}^{3}$ with $b \otimes m$ unique and a unique $\sigma \in \mathbb{R}$ such that

$$
\begin{aligned}
& N_{1}=-\sigma a \otimes n+b \otimes m \\
& N_{2}=(1-\sigma) a \otimes n+b \otimes m .
\end{aligned} \quad \text { and }
$$

But we do not yet know in (5.17) that $0<\sigma<1$.

Case 1 Assume that $\xi \| \eta$ for some $\zeta \in C_{0}^{\infty}(\Omega)$. Write $\eta=\tau \xi$ for some real $\tau$ with $\tau \neq-1$ by (5.16). Setting this in (5.15) with $c$ chosen such that $c \cdot \xi=1$, we obtain

$$
N_{1}+\tau N_{2}=\left(N_{1}+\tau N_{2}\right) c \otimes \xi=b \otimes m
$$

is rank one. We may now solve (5.11) and (5.18) to deduce (5.17) with $\sigma=\frac{\tau}{1+\tau}$ and

$$
\mathrm{m}=\int \chi \mathrm{E} \nabla \zeta \mathrm{dx}=\xi+\eta \text {. }
$$

The choice of $m$ is not unique; it may be any vector proportional to $\xi+\eta$.

Case 2 Suppose that $\xi$ is not parallel to $\eta$. Choosing $c \in R^{3}$ such that $c \cdot \xi=1$ and $c \cdot \eta=0$, then 


$$
N_{1}=N_{1} c \otimes \xi+N_{2} c \otimes \eta=p \otimes \xi+q \otimes \eta .
$$

Similarly,

$$
N_{2}=N_{1} c^{\prime} \otimes \xi+N_{2} c^{\prime} \otimes \eta=q^{\prime} \otimes \xi+r \otimes \eta .
$$

Substituting (5.19) and (5.20) into (5.15) yields that

$$
\left(q-q^{\prime}\right) \otimes \eta(c \cdot \xi)=\left(q-q^{\prime}\right) \otimes \xi(c \cdot \eta),
$$

whence $q=q^{\prime}$. Since $N_{2}-N_{1}=a \otimes n$,

$$
(q-p) \otimes \xi+(r-q) \otimes \eta=a \otimes n .
$$

Since $\xi$ is not parallel to $\eta$, it is necessarily the case that $q-p\|r-q\| a$. Let us write $q=$ $\mathrm{p}+\mathrm{ta}$ and $\mathrm{r}=\mathrm{q}+\mathrm{sa}=\mathrm{p}+(\mathrm{s}+\mathrm{t}) \mathrm{a}$ so

$$
N_{1}=p \otimes \xi+(p+t a) \otimes \eta \quad \text { and } \quad N_{2}=(p+t a) \otimes \xi+(p+(s+t) a) \otimes \eta(5.21)
$$

Note that $p$ is not parallel to a and $t \neq 0$ because rank $N_{1} \geq 2$. Now

$$
(1-\mu) N_{1}+\mu N_{2}=(p+\mu t a) \otimes \xi+(p+(\mu s+t) a) \otimes \eta
$$

which is rank one if and only if $p+\mu t a=p+(\mu s+t) a$, whence

$$
\mu=\frac{t}{t-s} \text { and } p+\mu t a=p+(\mu s+t) a=p+\frac{t^{2}}{t-s} a \text {. }
$$

With this choice of $\mu$,

$$
(1-\mu) N_{1}+\mu N_{2}=\left(p+\frac{t^{2}}{t-s} a\right) \otimes(\xi+\eta)=b \otimes m .
$$

From (5.11) and (5.22) we again obtain (5.17) with, for example,

$$
\mathrm{m}=\int \chi \mathrm{E} \nabla \zeta \mathrm{dx}=\xi+\eta
$$


To show uniqueness, suppose that for some $\sigma, b$, and $\mathrm{m}$,

Then

$$
N_{1}=-\sigma a \otimes n+b \otimes m
$$

$$
\operatorname{adj}\left(N_{1}+\sigma a \otimes n\right)=0 .
$$

The left hand side of this equation is linear in $\sigma$ with nonzero slope. Thus $\sigma$ is uniquely determined and so is $\mathrm{b} \otimes \mathrm{m}$.

The implication of the uniqueness is that the mapping

$$
\zeta \rightarrow \int_{\Omega} \chi \mathrm{E} \nabla \zeta \mathrm{dx}=\xi+\eta
$$

has a one dimensional range. Since the formula (5.23) provides us intuitively with the direction of the normal to $\mathrm{E}$, we see that $\partial \mathrm{E}$ is in some sense planar.

Step 4 There are real valued Lipschitz functions of a single variable $g$ and $f$ such that

$$
z(x)=g(x \cdot n) a+f(x \cdot m) b .
$$

Substituting the reperesentation (5.17) into the formula (5.10), with $\lambda_{2}=\lambda$ and $\lambda_{1}=1-\lambda$,

$$
\nabla z=\chi E(\lambda-\sigma) a \otimes n+\chi E b \otimes m .
$$

Choose a vector $c$ with $c \cdot a=0$ and $c \cdot b=1$, possible since rank $N_{1}>1$. Then for $\mathrm{u}(\mathrm{x})=\mathrm{c} \cdot \mathrm{z}(\mathrm{x})$,

$$
\nabla \mathbf{u}=\chi \mathbf{E m},
$$

and hence, since $\Omega$ is convex, there is a Lipschitz function $f(t)$ such that

$$
u(x)=f(x \cdot m) .
$$

In addition, we may write the set $\mathrm{E}$ as the cartesian product of two dimensional planes with an interval of the $\mathrm{m}$ - axis. More precisely, there is a (measurable) subset $I \subset \mathbb{R}$ such that 


$$
E=\{x \in \Omega: x \cdot m=\alpha, \alpha \in I\}
$$

Similarly, choosing $c^{\prime} \in \mathbb{R}^{3}$, with $c^{\prime} \cdot a=1$ and $c^{\prime} \cdot b=0, w(x)=c^{\prime} \cdot z(x)$ has the property

$$
\nabla w=\chi_{E}(\lambda-\sigma) n
$$

hence

$$
w(x)=g(x \cdot n) .
$$

Step 5

Equation (5.3) holds with $\sigma \in(0,1)$ and $\lambda(x) \equiv \sigma$ in a "propagation region."

Note that $\sigma \neq 0,1$ since $N_{i}$ have rank two. The assumption that $E$ is not of full measure means, by Fubini's Theorem for example, that there is a set

$$
\Sigma=\left\{x \in \Omega: x \cdot m=\alpha_{0}\right\}, \text { for some } \alpha_{0} \notin I,
$$

such that almost every $x \in \Sigma$ is a Lebesgue point of $\nabla z$ and $\Omega-E$. Hence $\nabla z=0$ in $\Sigma$ by (5.25) and $z=$ const. in $\Sigma$. We may assume that $z=0$ in $\Sigma$. Thus

$$
0=g(x \cdot n) a+f\left(\alpha_{0}\right) b \text { in } \Sigma
$$

Since $\mathrm{a}$ and $\mathrm{b}$ are independent,

$$
g(x \cdot n)=f\left(\alpha_{0}\right)=0 \quad \text { in } \Sigma .
$$

Let us set

$$
D=\{x \in \Omega: x \text { lies on a plane } x \cdot n=\beta \text { which intersects the plane } \Sigma\} \text {, }
$$

which we call a propagation region for $\Sigma$. From (5.27) we have that $g(x \cdot n)=0$ in $D$, hence

$$
\nabla \mathrm{g}=(\lambda-\sigma) \chi_{\mathrm{E}}=0 \quad \text { in } \mathrm{D} \text {. }
$$

Thus $\lambda=\sigma$ in $E \cap D$ a.e., so (i) $\sigma \in(0,1)$ and (ii) $\lambda=\sigma=$ const. in $E \cap D$ a.e. 


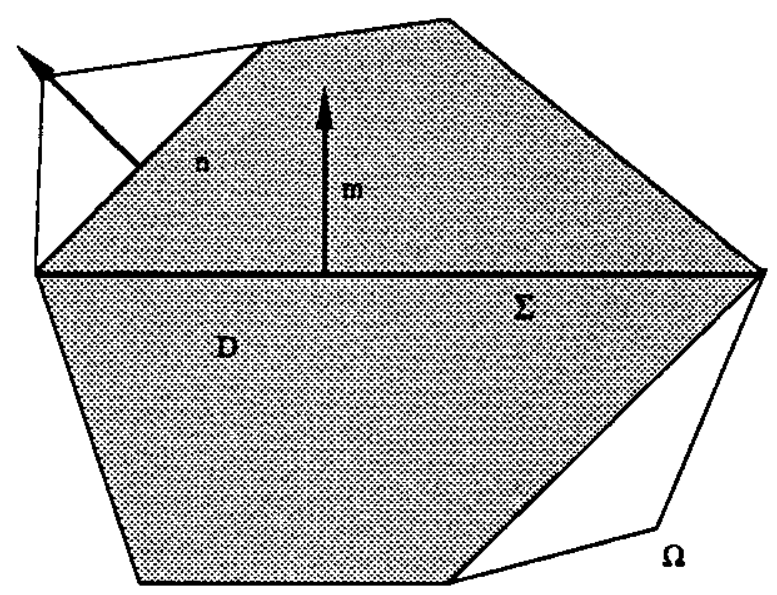

Fig. 10 Sketch of the propagation region $D$ of $\Sigma$.

Inside the propagation region of $\Sigma$, there may be a mix of martensite and austenite but the martensite occurs with fixed variant proportion $\sigma$, cf. Fig. 10. Outside the propagation region of $\sum$, there may be pure martensite, if such a domain does not intersect with any other propagation region, possibly of varying proportions, but usually a single variant, cf. Fig. 11. So, for example, under the assumption (5.8), we may write that the Young measure (5.4)

$$
v_{\mathrm{x}}=\chi \mathrm{E}\left((1-\sigma) \delta_{\mathrm{M}_{1}}+\sigma \delta_{\mathrm{M}_{2}}\right)+(1-\chi \mathrm{E}) \delta_{\mathrm{C}}, \quad \mathrm{x} \in \mathrm{D}
$$

An analogous representation of the Young measure may be given under the assumptions of Step 1. In this case,

$$
\begin{aligned}
& \lambda \equiv \lambda_{3}=\lambda_{3}(\mathrm{x} \cdot \mathrm{m}) \quad \text { and } \\
& v_{\mathrm{x}}=(1-\lambda)\left((1-\sigma) \delta_{\mathrm{M}_{1}}+\sigma \delta_{\mathrm{M}_{2}}\right)+\lambda \delta_{\mathrm{C}}, \quad \mathrm{x} \in \mathrm{D},
\end{aligned}
$$

where the propagation region is determined in a fashion analogous to (5.28). We have not observed this configuration in the laboratory, but it remains a mathematical possibility. 


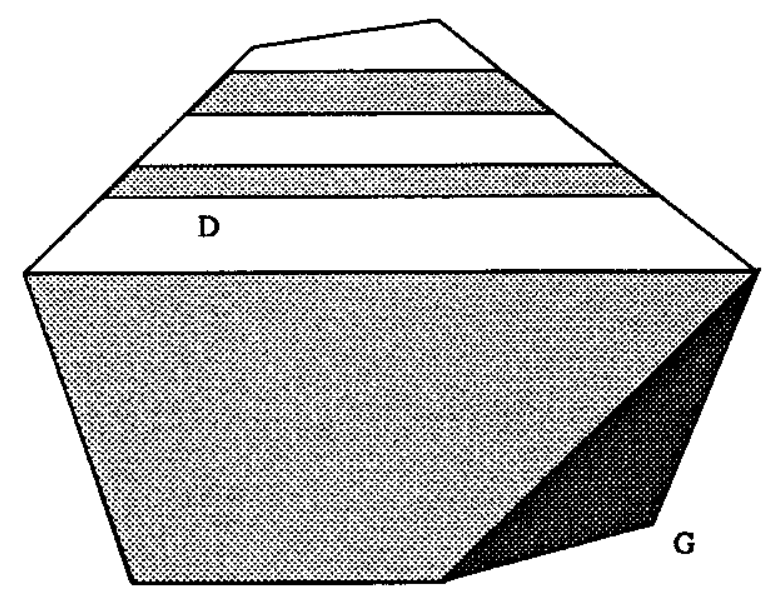

Fig. 11 In D there is a mixture of anstenite and finely twinned martensite and the latter occurs with fixed variant proportions. In $G$ there is pure martensite.

The condition (5.3) is exactly the condition that is the basis of the crystallographic theory of martensite presented, for example, by Christian [16]. For the origins of the theory, see Wechsler, Lieberman, and Read [73]. When the matrices $M_{i}$ and $C$ are only restricted to lie in potential wells, (5.3) gives rise to an algebraic problem: Given $R \in S O(3)$ with $R U_{2}-U_{1}=$ $\mathrm{a} \otimes \mathrm{n}$, find $\mathrm{Q} \in \mathrm{SO}(3), \mathrm{b}, \mathrm{m} \in \mathbf{R}^{3}$, and $\sigma \in(0,1)$ such that

$$
Q-\left[\sigma R U_{2}+(1-\sigma) U_{1}\right]=b \otimes m \text {. }
$$

An existence theorem for (5.30) is given in [6], not restricted to the cubic/tetragonal transformation. To summarize briefly what is known in the cubic/tetragonal case, $\mathrm{m}$, the reference normal to the austenite/martensite interface, turns out irrational but near a member of the family of $\{110\}$ planes, if $U_{1}$ is near 1 , and the angle between $m$ and $n$ is nearly $60^{\circ}$, both of which are observed experimentally. Although for one kind of microstructure, our approach and the Crystallographic Theory of Martensite are in complete agreement, the crystallographic theory is not based on energy considerations but on a kinematic condition known as the double shear mechanism. We think that a distinct advantage of our approach is that we are able to discuss general microstructures. To explore its general usefulness, additional predictions of the theory which may be made subject to experimental test are described in [7].

The examples of the preceding two sections illustrate how the behavior of minimizers of a variational principle may be reduced to questions of an algebraic character by the introduction of an 
appropriate parametrized measure. In addition to the papers already cited, ideas used in such analyses may be found in Matos [50] and Pedregal [56].

Acknowledgements We wish to thank our collaborators John Ball and Michel Chipot. We also wish to acknowledge many conversations with Jerry Ericksen and Mitchell Luskin.

\section{References}

1. Ball, J. M. 1976 Constitutive equations and existence theorems in nonlinear elastostatics, HeriotWatt Symp., I, (R. Knops,ed.) Pitman, 187-241

2. Ball, J. M. 1980 Strict convexity, strong ellipticity, and regularity in the calculus of variations, Math Proc Camb Phil Soc, 87, 501-513

3. Ball, J. M. 1984 Singular minimizers and their significance in elasticity, Phase Transformations and Material Instabilities in Solids, (Gurtin, M., ed) Academic Press, 1-20

4. Ball, J. M. 1989 Sets of gradients with no rank-one connections (to appear)

5. Ball, J. M. 1989 A version of the fundamental theorem for Young measures, these proceedings

6. Ball, J. M. and James, R. 1987 Fine phase mixtures as minimizers of energy, Arch. Rat Mech. Anal.,100, 15-52

7. Ball, J. M. and James, R. 1989 Proposed experimental tests of a theory of fine microstructure and the two well problem (to appear)

8. Ball, J. M. and Murat, F. $1984 \mathrm{~W}^{1, p}$ - quasiconvexity and variational problems for multiple integrals, J. Fnal Anal, 58,225-253

9. Ball, J. M., and Knowles, G. 1986 Liapunov functions for thermomechanics with spatially varying boundary temperatures, Arch. Rat. mech. Anal., 92, 193-204

10. Basinski, Z. S. and Christian, J. W. 1954 Experiments on the martensitic transformations in single crystals of indium-thallium alloys, Acta Met. 2, 149-166

11. Battacharya, K 1989 Thesis, University of Minnesota

12. Burkart, M. W. and Read, T. A. 1953 Diffusionless phase change in the indium-thallium system, J. of Metals, 5, 1516-1524

13. Chipot, M. and Evans, L. C. 1986 Linearisation at infinity and Lipschitz estimates for certain problems in the calculus of variations, Proc. R. Soc. Edin., 102A, 291-303

14. Chipot, M. and Kinderiehrer, D. 1988 Equilibrium configurations of crystals, Arch. Rat. Mech. Anal. $103,237.277$

15. Chipot, M., Kinderiehrer, D., and Vergara-Caffarelli, G. 1986 Smoothness of linear laminates, Arch. Rat. Mech. and Anal. 96, 81-96

16. Christian, J. W. 1975 The theory of transformations in metals and alloys, Pergamon 
17. Collins, C. and Luskin, M. 1989 The computation of the austenitic-martensitic phase transition (these procedings)

18. Dacorogna, B. 1982 Weak continuity and weak lower semicontinuity of nonlinear functionals, Springer Lecture Notes 922 (1982)

19. Dacorogna, B. and Fusco, N. 1985 Semi-continuite des fonctionneiles avec contraintes du type "det grad u > 0", Boll. U M I, 6, 179-189

20. Eftis, J., MacDonald, D. E., and Arkilic, G. M. 1971 Theoretical calculation of the pressure variation of second-order elastic coefficients for alkali metals, Mater. Sci. Eng. 7, 141-150

21. Ericksen, J. L. 1973 Loading devices and stability of equilibrium, in Nonlinear Elasticity, Academic Press, 161-173

22. Ericksen, J. L. 1977 Special topics in elastostatics, Adv, in appl. mechanics, (C.-S. Yih, ed.) Academic Press 7, 189-243

23. Ericksen, J. L. 1979 On the symmetry of deformable crystals, Arch. Rat. Mech. Anal. 72, 1-13

24. Ericksen, J. L. 1980 Some phase transitions in crystals, Arch. Rat. Mech. Anal. 73, 99-124

25. Ericksen, J. L. 1981 Some simpler cases of the Gibbs phenomenon for thermoelastic solids, J.of thermal stresses, $4,13-30$

26. Ericksen, J. L. 1983 IIl posed problems in thermoelasticity theory, Systems of Nonlinear Partial Differential Equations, (Ball, J., ed) D. Reidel, 71-95

27. Ericksen, J. L. 1984 The Cauchy and Bom hypotheses for crystals, Phase Tranformations and Material Instabilities in Solids, (Gurtin, M., ed) Academic Press, 61 -78

28. Ericksen, J. L. 1986 Stable equilibrium configurations of elastic crystals, Arch. Rat. Mech. Anal. 94, 114

29. Ericksen, J. L. 1987 Twinning of crystals I, Metastability and Incompletely Posed Problems, IMA Vol. Math. Appl. 3,(Antman, S., Ericksen, J.L., Kinderlehrer, D., Müller, I.,eds) Springer, 77-96

30. Ericksen, J. L. 1988 Some constrained elastic crystals, Proc. Symp. Material instabilities in continuum mechanics, Heriot-Watt (Ball, J. M. ed.) Oxford, 119-136

31. Fonseca, I. 1985 Variational methods for elastic crystals, Arch. Rat. Mech. Anal., 97, 189-220

32. Fonseca, I. 1988 The lower quasiconvex envelope of the stored energy function for an elastic crystal, J. Math. pures et appl, 67, 175-195

33. Fonseca, I. 1989 Interfacial energy and the Maxwell rule, Arch. Rat. Mech. Anal.

34. Gibbs, J. W. 1961 On the equilibrium of heterogeneous substances, The Scientific Papers of J. Willard Gibbs, Vol 1., Dover

35. Gurtin, M. E. 1983 Two phase deformations of elastic solids, Arch. Rat. Mech. Anal., 84, 1-29

36. Gurtin, M. E. 1986 On phase transitions with bulk, interfacial, and boundary energy, Arch. Rat. Mech. Anal., 96, 243-264

37. Herring, C. 1951 Some theorems on the free energy of crystal surfaces, Phys. Rev. 82, 87-93

38. James, R. D. 1986 Displacive phase transformations in solids, J. Mech. Phys. Solids, 34, 359-394 
39. James, R. D. 1986 Phase transformations and non-elliptic free energy, New Perspectives in Thermodynomics (Serrin, J., ed) Springer, 223-239

40. James, R. D. 1987 The stability and metastability of quartz, Metastability and Incompletely Posed Problems, IMA Vol. Math. Appl. 3,(Antman, S., Ericksen, J.L., Kinderlehrer, D., Müller, I.,eds) Springer, 147-176

41. James, R. D. 1988 Microstructure and weak convergence, Proc. Symp. Material instabilities in continuem mechanics, Heriot-Watt (Ball, J. M. ed.) Oxford, 175-196

42. Kinderlehrer, D. 1987 Twinning in crystals II, Metastability and Incompletely Posed Problems, IMA Vol. Math. Appl. 3,(Antman, S., Ericksen, J.L., Kinderlehrer, D., Muller, I.,eds) Springer, 185211

43. Kinderlehrer, D. 1988 Remarks about the equilibrium configurations of crystals, Proc. Symp. Material instabilities in continuum mechanics, Heriot-Watt (Ball, J. M. ed.) Oxford, 217-242

44. Kinderiehrer, D. and Vergara-Caffarelli, G. The relaxation of functionals with surface energies, to appear

45. Kohn, R. V. and Strang, G. 1987 Optimal design and relaxation of variational problems, I,CPAM $34,113-137$

1987 Optimal design and relaxation of variational problems, II,CPAM

46. Kohn, R. V. and Strang, G. $34,139-182$

47. Kohn, R. V. and Strang, G. 1987 Optimal design and relaxation of variational problems, III,CPAM $34,353-377$

48. Marcellini, P. 1986 On the definition and the lower semicontinuity of certain quasiconvex integrals, Ann. Inst. H. Poincaré; Analyse non linéaire, 3, 391-409

49. Mascolo, E. and Schianchi, R. 1983 Existence theorems for non convex problems, J. Math pures et appl, 62,(1983), 349-359

50. Matos, J. 1989 Thesis, University of Minnesota

51. Morrey, C. B., Jr. 1966 Multiple Integrals in the Calculus of Variations, Springer

52. Maller, I. 1979 A model for a body with shape memory, Arch. Rat. Mech. Anal., 70, 61-77

53. Müller, I. and Wilmanski, K. 1980 A model for phase transition in pseudoelastic bodies, D Nuovo Cim., 57B, 283-318

54. Parry, G. 1981 On phase transitions involving intemal strain, Int. J. Solids Structures, 17, 361378

55. Parry, G.

On shear bands in unloaded crystals, to appear

56. Pedregal, $\mathbf{P}$.

Thesis, University of Minnesota

57. Pego, R.

1985 Phase transitions in one dimensional nonlinear viscoelasticity: admissibility and stability, Arch. Rat. Mech. Anal. 97, 353-394

58. Pego, R. 1987 Phase transitions in one dimensional nonlinear viscoelasticity: admissibility and stability, Dynamical Problems in Continuum Physics, I M A Vol. Math. Appl. 4 (Bona, J., Dafermos, C., Ericksen, J. L., and Kinderlehrer, ., eds)277-288

59. Pipkin, A. C. 1986 The relaxed energy density for isotropic elastic membranes, IMA J Appl Math, 36, 
60. Pippard, A. B. 1957 The elements of classical thermodynamics, Cambridge

61. Pitteri, M. 1985 On v + 1 lattices, J. Elasticity, 15, 3-25

62. Pitteri, M. 1986 On type-2 twins in crystals, Int. J. Plasticity, 2, 99-106

63. Pitteri, M. 1987 A contribution to the description of natural states for elastic crystalline solids, Metastability and Incompletely Posed Problems, IMA Vol. Math Appl. 3,(Antman, S., Ericksen, J.L., Kinderlehrer, D., Müller, I.,eds) Springer, 295-310

64. Reshetnyak, Yu. G. 1967 Liouville's theorem on conformal mappings under minimal regularity assumptions, Siberian Math J., 8, 631-653

65. Reshetnyak, Yu. G. 1967 On the stability of conformal mappings in multidimensional spaces, Siberian Math J., 8, 69-85

66. Simpson, H. C. and Spector, S. J. 1987 On the sign of the second variation in finite elasticity, Arch. Rat. Mech. Anal. 98, 1-30

67. Slemrod, M. 1986 Interrelationships among mechanics, numerical analysis, compensated compactness, and oscillation theory, Oscillation Theory, Computation, and Methods of Compensated Compactness, I M A Vol. Math. Appl. 2, (Dafermos, C., Ericksen, J. L, 309-336. , Kindertehrer, D., and Slemrod, M. eds)

68. Tartar, L. 1979 Compensated compactness and applications to partial differential equations, Nonlinear analysis and mechanics; Heriot-Watt symp. IV, (Knops, R. J. ed) Pitman, (1979), 136-212

69. Tartar, L. 1983 The compensated compactness method applied to systems of conservation laws, Systems of nonlinear partial differential equations (Ball, J. M., ed) Riedel, 263-285

70. Tartar, L. 1984 Étude des oscillations dans les équations anx dérivées partielles nonlinéaires, Springer Lect. Notes Physics, 195, 384-412

71. Tisza, L. 1951 On the general theory of phase transformations in solids, (Smoluchowski, R, Mayer, J. E., and Weyl, W. A., eds) Wiley, 1-35

72. van Tendelo, G., van Landuyt, J., and Amelinckx, S. 1976 The $\alpha-\beta$ phase transition in quartz and AlPo4 as studied by electron microscopy and diffraction. Phys. Stat. Sol., a33, 723-735

73. Wechsler, M. S., Lieberman, D. S., and Read, T. A. 1953

On the theory of the formation of martensite, Trans. AIME J. Metals, 197, 1503-1515

74. Young, L. C. 1969 Lectures on calculus of variations and optimal control theory, W.B. Saunders 